\title{
CHACRAS DISPERSAS. UNA APROXIMACIÓN ETNOGRÁFICA Y ARQUEOLÓGICA AL ESTUDIO DE LA AGRICULTURA PREHISPÁNICA EN LA REGIÓN SERRANA DE CÓRDOBA (ARGENTINA).
}

\author{
Matías E. Medina y Sebastián Pastor \\ Cátedra de Prehistoria y Arqueología (U.N.Cba.), CONICET.
}

\section{Resumen}

Las comunidades productoras de alimentos que ocuparon las Sierras de Córdoba (Argentina) entre ca. 1200-300 AP implementaron una estrategia predominante agrícola, con estrategias complementarias de caza-recolección y, probablemente, pastoriles. Sin embargo, el conocimiento arqueológico acerca de las prácticas agrícolas prehispánicas es actualmente limitado y está parcialmente construido en base a líneas de evidencia indirectas. La información más significativa proviene de la documentación producida durante los primeros momentos del régimen colonial español (siglos XVI y XVII), que describe un sistema agrícola nativo sin regadios, con uso de numerosas chacras. En este trabajo se presenta información etnográfica actual sobre la producción agrícola de pequeña escala en diferentes ámbitos de las Sierras de Córdoba, colocando especial énfasis en el sistema de dispersión de campos de cultivos y en cómo éste permite afrontar algunas situaciones de riesgo que se imponen sobre la actividad. Se derivan luego algunas expectativas arqueológicas y se presenta información actualizada acerca de la distribución espacial de sitios prehispánicos. Finalmente, se plantea la posibilidad de que algunas de las situaciones arqueológicas reconocidas en el Valle de Salsacate, en la porción septentrional del Valle de Punilla y en sectores específicos de las pampas de altura tengan relación con la implementación de este tipo de estrategias.

Palabras claves: Sierras de Córdoba - prácticas agrícolas prehispánicas - etnografía- riesgo

\begin{abstract}
The prehispanic agricultural groups who occupied Córdoba Hills (ca. 1200-300 BP) established a predominant agricultural strategy, with hunting-gathering and possibily pastoral like complementary strategies. Howewer, the present archaeoleogical knowledge about prehispanic agricultural practices is very limited and it is parcially built on the basis of indirect evidence. The most important data comes from the first moments of the spaniard colonial regime etnohistoric documentation (XVII century), which describes a non irrigated agricultural system with the use of scattering fields. Present etnographic information about small scale agricultural production in different localities of Córdoba Hills, with special emphasis on field scattering like risk adverse strategies, is shown in this paper. Archaeological consequences and new data of archaeological sites space distribution are presented. Finally, the possibility that some archaeological situations from Salsacate valley, northern Punilla valley and specific settings in montane grassland belt, bear relation to field scattering strategies, is considered.
\end{abstract}

Key word: Córdoba Hills - prehispanic agricultural practices - ethnography - risk

\section{INTRODUCCIÓN}

Las comunidades productoras de alimentos que habitaron las Sierras de Córdoba (Argentina) entre ca. 1200-300 AP desarrollaron una estrategia predominante agrícola, complementada con actividades de caza y recolección (Berberián 1999; Berberián y Roldán 2001). Recientes estudios osteométricos realizados sobre huesos de camélidos indican la presencia y posible manejo de animales domésticos (i.e. Lama glama, Pastor y Medina 2003a). 
Sin embargo, el conocimiento arqueológico acerca de las prácticas agrícolas prehispánicas es actualmente limitado y está parcialmente construido en base a líneas de evidencia indirectas. Entre ellas pueden citarse la presencia de posibles herramientas para el laboreo agrícola (azuelas), de instrumentos de molienda (morteros y conanas) y la ubicación de las bases residenciales en terrenos potencialmente cultivables (Pastor 2002a).

La información más significativa proviene de la documentación producida durante el régimen colonial español, instalado en la segunda mitad del siglo XVI, la que aporta datos acerca de las prácticas agrícolas implementadas por la población aborigen. Sintéticamente, las fuentes etnohistóricas describen un sistema agrícola sin regadíos (salvo una excepción en la zona noroeste de las sierras), basado en el cultivo de maíz, zapallo, poroto, quínoa, maní y camote, con uso de numerosas chacras ubicadas en los alrededores de las bases residenciales y en cañadas y faldeos de los cerros (Bixio y Berberián 1984; Piana de Cuestas 1992).

A partir del desarrollo de investigaciones arqueológicas de escala regional en el sur del Valle de Punilla se identificó la presencia de sitios grandes al aire libre, ubicados en extensos piedemontes y fondos de valle, que fueron interpretados como asentamientos residenciales y agrícolas. Asimismo, se comprobó la existencia de otros sitios a cielo abierto de pequeñas dimensiones, localizados en quebradas tributarias, que presentan una baja densidad de materiales arqueológicos (Berberián y Roldán 2001; Roldán 1998; Roldán y Pastor 1997). Estos sitios fueron considerados "campos de cultivo de reducidas dimensiones" (Berberián y Roldán 2001), que formaban parte de una estrategia dirigida a minimizar los riesgos y a otorgar seguridad a la producción agrícola.

Otras consideraciones sobre la agricultura prehispánica en las Sierras de Córdoba derivan de las investigaciones realizadas en la cuenca del Río Copacabana, en el sector noroeste de la provincia. En una serie de cálculos dirigidos a estimar la densidad demográfica prehistórica y a establecer el aporte relativo de las diferentes actividades económicas, se asume una explotación agrícola "máxima y óptima", con un aprovechamiento de la totalidad de terrenos cultivables (Laguens 1999).

En este trabajo se presenta información etnográfica actual acerca de las características de la agricultura de pequeña escala en las Sierras de Córdoba, colocando especial énfasis en las estrategias utilizadas para enfrentar los factores de riesgo que afectan dicha actividad, muchos de los cuales también estaban presentes, según información etnohistórica, durante los siglos XVIy XVII.

En segundo lugar, se aporta nueva información sobre la distribución espacial de sitios prehispánicos. De acuerdo al patrón espacial de la agricultura serrana actual, se plantea la posibilidad de que algunas situaciones reconocidas en la porción septentrional del Valle de Punillla y en el Valle de Salsacate tengan relación con la implementación de estrategias de dispersión de campos de cultivo.

Por otra parte, a partir de un caso etnográfico registrado en el ambiente serrano de altura (Pampa de Achala), tradicionalmente considerado inapropiado para la actividad agrícola, se analizan las condiciones bajo las cuales se practica esta actividad en el presente y se describen situaciones arqueológicas que podrían indicar la presencia de cultivos de pequeña escala en época prehispánica. 


\section{EL RIESGO AGRÍCOLA EN LAS SIERRAS DE CÓRDOBA}

Según Cashdan (1990), el riesgo designa variaciones impredecibles en las condiciones ecológicas y económicas, mientras que la incertidumbre alude a la falta de información acerca de dichas fluctuaciones. Siguiendo a Bousman (1993), se prefiere no diferenciar entre riesgo e incertidumbre, quedando ambos conceptos englobados bajo el término "riesgo".

En las Sierras de Córdoba muchos factores de riesgo pueden afectar a los campos de cultivo. Entre ellos se pueden mencionar: 1) variabilidad e impredecibilidad anual de las precipitaciones; 2) tormentas de granizo; 3) heladas; y 4) plagas dañinas (langostas, gusanos, catas, etc.).

Se utilizará para su descripción información geográfica actual y etnohistórica. Debe destacarse la importancia de esta última fuente, ya que señala que estos factores, actuando de manera aislada o en combinación, eran responsables del fracaso de una de cada tres cosechas durante los primeros años del asentamiento español (Piana de Cuestas 1992; Roldán 1998).

\section{El régimen de precipitaciones}

En el sector central de Sierras Centrales (sensu Berberián 1999), las lluvias se concentran entre Septiembre y mediados de Marzo y, en condiciones normales, son adecuadas para las demandas productivas (Roldán 1998). Sin embargo, este régimen se caracteriza por su irregularidad interanual y microregional (Capitanelli 1979). A modo de ejemplo, para la primera parte del siglo XX se citan como "años secos" a 1937, 1938 y 1946, con precipitaciones que alcanzaron respectivamente los 400, 400, $493 \mathrm{~mm}$. Por otra parte, los años 1931, 1943 y 1956 fueron considerados lluviosos, con precipitaciones de 904, 863 y $937 \mathrm{~mm}$ (Terzaga 1963).

Según la Estación Córdoba Aeropuerto del Servicio Meteorológico Nacional (SMN), la media de precipitaciones para las proximidades de la ciudad de Córdoba en los últimos 30 años es de $836 \mathrm{~mm}$ (Medina 2002). Sin embargo, el registro de precipitaciones muestra un patrón poco predecible, con gran variación anual. Para el caso de los años 1983 a 2000 la media de precipitaciones es de $859 \mathrm{~mm}$, pero la variabilidad anual alcanza un desvío estándar de $240 \mathrm{~mm}$ (Medina 2002).

Esta variabilidad se ve acentuada si se consideran los niveles de concentración estacional. Para el caso de los meses de Septiembre y Octubre, que es cuando preferentemente se produce la siembra, la media de precipitaciones para los mismos años es de $112 \mathrm{~mm}$, con un desvío estándar de $64 \mathrm{~mm}$. Es decir que en ocasiones las lluvias apenas alcanzan los $50 \mathrm{~mm}$ en esos dos meses.

Otro elemento a tener en cuenta es que la cantidad de lluvias varía significativamente entre los distintos sectores de las sierras. En efecto, los valles orientales (Punilla, Los Reartes, Calamuchita) son más lluviosos que los occidentales (Salsacate, Tránsito) y que las Sierras del Norte (Capitanelli 1979).

Esta marcada impredecibilidad del régimen de precipitaciones es también destacada por las fuentes documentales de los siglos XVI y XVII. En efecto, durante los primeros años del asentamiento español (1573-1620) un $17.5 \%$ de los años fueron afectados por sequías, mientras que en un 13 \% se registraron inundaciones (Piana de Cuestas 1992; Roldán 1998). 


\section{Granizo}

Otro importante factor de riesgo son las tormentas de granizo, muy frecuentes en las sierras de Córdoba durante el verano (Hordij et al. 1996; Papadakis 1956). Si bien su acción tiene un carácter marcadamente localizado, pueden producir importantes daños en los cultivos, como la rotura de tallos y hojas, seguida por la aparición de podredumbre y enfermedades foliares que conducen a la pérdida de las plantas afectadas (López Bellido 1991).

Estas fuertes tormentas de granizo, y las consecuencias sobre la producción agrícola, fueron descriptas con frecuencia en la documentación de la época colonial. Por ejemplo, en el año 1616 el Cabildo de la ciudad de Córdoba propuso la organización de festejos en el día de San Pedro Mártir, abogado de truenos, rayos y tempestades, ya que "todos los años en muchas partes de los terminos desta ciudad cae piedra granizo y rayos y otras tempestades" (Piana de Cuestas 1992).

\section{Heladas}

Las heladas son frecuentes en casi toda la Provincia de Córdoba (Papadakis 1956). En los sectores elevados de las sierras comienzan en Marzo, mientras que en los valles se dan normalmente un mes más tarde, para finalizar luego en Noviembre y Octubre, respectivamente (Capitanelli 1979).

Se trata de un fenómeno muy localizado, ya que afecta con preferencia las zonas topográficamente bajas, como los fondos de valle, mientras que se manifiestan con menor intensidad en las laderas de los cerros y quebradas laterales.

Debe considerarse que el impacto de este fenómeno sobre la producción agrícola depende de la fecha de ocurrencia. Las heladas más perjudiciales no son aquellas que se producen dentro del período normal, sino las más tempranas o tardías. Por ejemplo, si ocurren en primavera cuando las plantas se encuentran desarrollando sus brotes tiernos o con pocos días de vida activa, el daño de la helada será mayor (SMN 1985).

En el caso específico del maíz, una helada tardía puede ocasionar la muerte de las plantas cuando el punto de crecimiento se encuentra por encima de la superficie del suelo. Por otro lado, las heladas tempranas, que se producen antes de que el grano de maíz esté maduro, también pueden causar pérdidas parciales o totales de las cosechas (López Bellido 1991).

\section{Plagas}

Las plagas de ortópteros, comúnmente llamados langostas (Rhomatocerus pictus, Dichroplus sp., Staurorhectus longicornis, etc.), son una importante amenaza para los cultivos en la Provincia de Córdoba (Bucher y Abalos 1979). Una de las características de estos insectos es que causan daños muy localizados, ya que sólo destruyen el sector del espacio en el cual la "manga" se posa. De esta manera, puede verse afectada a una chacra en particular y no una vecina localizada a escasa distancia.

De acuerdo a los informantes locales, en la actualidad la langosta no representa una gran amenaza como lo fue en el pasado, ya que ha sido prácticamente exterminada mediante el 
uso de pesticidas y fumigaciones a gran escala. Sin embargo, todos recuerdan los daños causados por las enormes "mangas" de langostas, que lograban "oscurecer el cielo".

La amenaza de las langostas es también destacada en las fuentes etnohistóricas. Refiriéndose al Tucumán en general, el cronista Bernabé Cobo (citado por Piana de Cuestas 1992) expresa que "la langosta... es muy perjudicial, como es en el reino de Chile y provincia de Tucuman, y Santa Cruz de la Sierra, a donde a tiempo se ven en tanta muchedumbre, que parecen nublados y talan los sembrados...".

\section{INFORMACIÓN ETNOGRÁFICA}

\section{La agricultura de pequeña escala en las Sierras de Córdoba}

La agricultura practicada en las Sierras de Córdoba, a excepción de la Pampa de Pocho, Pampa de Olaen y, en algunos casos, el Valle de Punilla y de Calamuchita, aún se basa en métodos tradicionales que implican la diversidad de cultivos y la dispersión de chacras. De esta manera, la población serrana logra satisfacer parte de sus necesidades básicas aún en condiciones adversas (suelos marginales, sequías, etc.) y sin depender de la mecanización ni del uso de fertilizantes o pesticidas.

Básicamente, se trata de una horticultura destinada al consumo doméstico y diseñada para optimizar la producción a largo plazo más que al corto plazo. El destino final de la cosecha no es el mercado sino la unidad doméstica, entendida como la unidad mínima de producción, donde es utilizada para satisfacer parte de la dieta familiar así como para complementar la alimentación de los animales domésticos. No obstante, debe considerarse que la economía familiar está muy diversificada, percibiendo ingresos de un amplio rango de actividades, como el pastoreo extensivo, trabajo asalariado y venta de artesanías en cuero o cestería de hojas de palma.

\section{Metodología}

Se realizaron una serie de entrevistas con pobladores que aún practican una agricultura de pequeña escala. Con el fin de cubrir todas las situaciones ambientales presentes en las sierras de Córdoba, se seleccionaron cinco unidades domésticas de distintos ámbitos de las sierras (valle, ecotono valle-llanura chaqueña y pampas de altura). Las entrevistas se realizaron paralelamente a los trabajos de campos, en distintas épocas del año, durante los años 2001-2004.

Para el ambiente de valle, se entrevistó a las de familias de Don Benito Arias, Don Marcos Oliva y Don Julian Cataldo, habitantes del paraje "Alto de la Cruz", en el sector occidental del Valle de Salsacate. Para el caso del ecotono valle-llanura chaqueña se entrevistó a Doña Rosa de López, quien habita el paraje conocido como "Vado de López", a unos 15 km. al sur de la localidad de San Marcos Sierras. Por último, se realizaron entrevistas con Don Jesús Reina, quien vive en los pastizales del norte de Pampa de Achala, a 1750 m.s.n.m. (Figura 1).

La entrevista consistió en una serie de preguntas abiertas básicas: ¿cuántas chacras posee?, ¿se cultivan todas al mismo tiempo?, ¿existen chacras más productivas que otras y qué factores intervienen?, ¿cuánto tiempo se mantienen productivas y cuánto tiempo de barbecho necesitan?. Asimismo, se preguntó qué especies y variedades se cultivan y cuáles son los factores de riesgo considerados, así como las estrategias desarrolladas para enfrentarlos. 


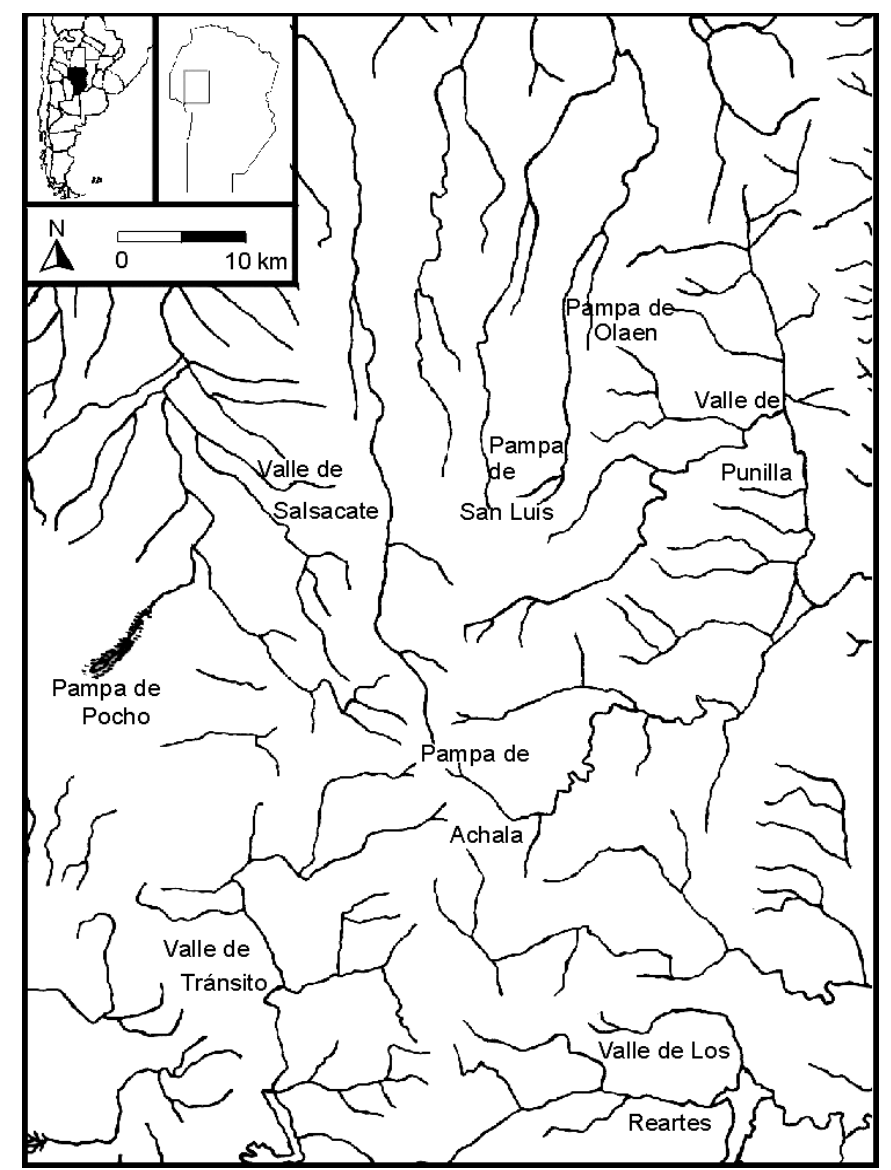

Figura 1. Ubicación de los ambientes de altura y valles circundantes

Las chacras fueron visitadas con los informantes, medidas y fotografiadas. Paralelamente, se ubicaron en la cartografía regional (escala 1:50000) y, cuando fue posible, en fotografías aéreas (escala 1:20000).

Para facilitar la presentación y posterior discusión, se decidió dividir los resultados en dos partes. Primero, se presentará la información obtenida para el caso del ambiente de valle y su ecotono con la llanura chaqueña. Posteriormente, se presentará un caso específico de agricultura con altos niveles de riesgo: Don Jesús Reina y sus pequeñas chacras en quebradas protegidas de las pampas de altura.

La agricultura en el ambiente de valle y sectores ecotonales

De acuerdo a la información obtenida durante las entrevistas, la agricultura serrana de pequeña escala puede caracterizarse de la siguiente manera:

1- Cada unidad doméstica trabaja durante la estación productiva un número variable de chacras no contiguas, cuyo tamaño rara vez supera las dos hectáreas. Las mismas se encuentran dispersas en distintos microsectores del paisaje local, en una gran diversidad de terrenos, como fondos de valle, terrenos abiertos, quebradas protegidas, 
bordes de los ríos, cañadas laterales, suelos arenosos y arcillosos, expuestos a mayor y menor radiación solar, etc. A modo de ejemplo, podemos ver la Figura 2, donde se observa que la familia de Don Benito Arias tiene una chacra en un terreno abierto de grandes dimensiones, aunque también utiliza otras ubicadas en cotas más altas, sobre cañadas laterales. En la misma figura se puede ver el caso de Don Marcos Oliva, quien utiliza dos chacras cercanas a su casa, en quebradas laterales, aunque debe rentar otra en el fondo de valle, en un sector conocido como el "bajo", a más de dos kilómetros de su casa y con un marcado desnivel del terreno.

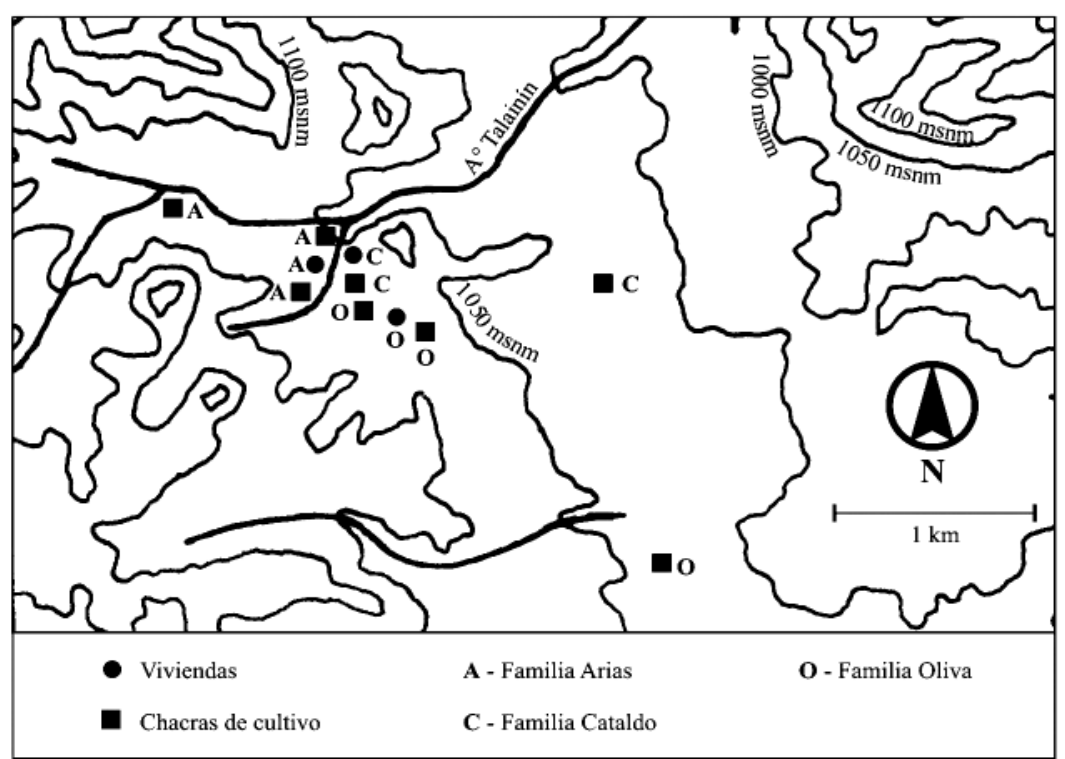

Figura 2. Patrón espacial de la agricultura de Cañada de Salas y Alto de la Cruz, Salsacate.

2- Como las tierras son roturadas mediante la utilización de un arado "mansero" tirado por un caballo, son las primeras lluvias de Octubre y Noviembre las que marcan el inicio del calendario agrícola. Al parecer, con esta tecnología es difícil arar sin la ayuda de las lluvias, ya que el terreno presenta un elevado nivel de dureza.

3- No todos los años se cultiva el mismo número de chacras. En años lluviosos se cultiva el mayor número de chacras posible. En cambio, en años secos se siembran menos, dada las dificultades para arar la tierra.

4- Siempre se comienza el ciclo agrícola arando la tierra considerada más productiva. Si las lluvias lo permiten, se trabaja el resto de las chacras. En consecuencia, no todas las chacras son roturadas al mismo tiempo.

5- Al no utilizarse fertilizantes, al cabo de cuatro o cinco años la productividad de la tierra comienza a agotarse. Esta situación lleva a rotar el uso de las chacras, alternando su utilización con períodos de barbecho de dos o tres años.

6- El principal cultivo es siempre el maíz, del cual se utiliza una amplia gama de subespecies, cada una con sus ventajas y desventajas. Es importante aclarar que todas estas variedades son cultivadas dentro de una misma chacra, separadas en ocasiones simplemente por surcos de arado. En la actualidad hemos registrado la utilización de los fenotipos "blanco", " amarillo", "pisingallo", "moro" y "pinchudo". Hasta hace pocos años también se aprovechaban otras variedades como "capia", 
"ankua", "overo" y "chanchero". Esta diversidad favorece que la cosecha del cereal llegue a buen término, ya que algunas variedades son más resistentes que otras (por ejemplo, el amarillo resiste mejor la sequía). Además, algunos fenotipos generan choclos y maíz más tempranamente (i.e. el "blanco"), facilitando la cosecha y evitando riesgos como los de las heladas tempranas.

7- También se utilizan variedades de zapallo ("angola", "anko" y "criollo") y porotos, cuya siembra combinada permite aprovechar al máximo los nutrientes del suelo, evitar las malezas e incrementar la producción. En algunos casos, se registró el cultivo pasturas (sorgo y alfalfa), sandías y melones.

8- El granizo es un elemento muy destructivo. Sin embargo, al tratarse de un fenómeno localizado, puede ocurrir que dañe a una chacra y no a la vecina. Por ejemplo en el caso del occidente del Valle de Salsacate, puede dañar una chacra del "bajo" (Cañada de Salas) y no una del "alto" (Alto de la Cruz), o viceversa. Cada tres o cuatro años la cosecha de una chacra se pierde por la acción de la "piedra".

9- En cuanto a las heladas tardías o tempranas, son altamente destructivas. Sin embargo, de acuerdo al modo en que actúan según las características microtopográficas, sólo afectan a los terrenos ubicados en los sectores deprimidos del "bajo". No suelen afectar a las chacras ubicadas en cotas elevadas, que no siempre son las más productivas.

10- Con respecto a las quebradas protegidas, estas parecen conservar mejor la humedad que los sectores abiertos expuestos a mayor radiación solar, razón por la cual se mantienen productivas aún en años secos.

11- La variación en la productividad de los cultivos no puede explicarse con referencia a factores de manejo (productividad de las tierras, agotamiento, densidad inadecuada de semillas sembradas, fecha de siembra, etc.). Los factores de riesgo mencionados son la causa principal de la varianza en los rendimientos. En todos los casos, la dispersión de los campos de cultivo permite a los agricultores locales manejar y reducir el riesgo de manera considerable, pero a expensas de una importante pérdida de la eficiencia.

12- Al utilizarse chacras dispuestas a distintas gradientes, suelos, exposición al sol, humedad, etc., sumado a que no todas son sembradas al mismo tiempo y a que se cultivan diversas especies y variedades, la cosecha puede realizarse a lo largo de un extenso período de tiempo. Esta situación permite un aprovechamiento más efectivo de la cosecha y de la mano de obra disponible.

\section{Don Jesús Reina y la agricultura en los ambientes serranos de altura}

Durante la realización de trabajos de prospección y excavación de sitios arqueológicos en las nacientes del Río Yuspe, en la porción norte de la Pampa de Achala, conocimos a Don Jesús Reina y su familia. El aspecto que logró sorprendernos en cuanto a sus actividades de subsistencia es que casi todos los años siembra maíz, zapallo y papa en dos pequeñas chacras próximas a su vivienda.

Tradicionalmente se consideró que la agricultura de pequeña escala era impracticable en los sectores altos de las Sierras de Córdoba, dada la pobreza de los suelos y, fundamentalmente, las duras condiciones climáticas. En efecto, de manera contrastante con lo que ocurre en los valles serranos, la temperatura media anual apenas alcanza en la actualidad $\operatorname{los} 10^{\circ} \mathrm{C}$., mientras que el período de heladas es más extenso, las nevadas 
son frecuentes en invierno e incluso primavera y los fuertes vientos azotan en forma regular.

La actividad agrícola desarrollada por Reina puede caracterizarse, de acuerdo a la información obtenida durante las entrevistas, de la siguiente manera:

1. Se utilizan dos pequeñas chacras no contiguas próximas a la vivienda, aprovechando espacios cerrados con pircas por los anteriores habitantes, quienes los utilizaban como corrales. Las superficies bajo cultivo son de apenas 50 y $100 \mathrm{~m}^{2}$.

2. Tanto la vivienda como las dos chacras se encuentran en el fondo de una quebrada protegida, a orillas del Río Yuspe. Si bien ambas chacras están a escasa distancia una de otra, existen diferencias en cuanto a la calidad de los suelos. La más alejada de la vivienda tiene menos cantidad de tierra y experimenta agotamientos con mayor frecuencia.

3. Las tierras son roturadas con uso de herramientas manuales y la siembra se produce al iniciarse las lluvias, entre Octubre y Noviembre. En los años en que las precipitaciones son escasas se riega con agua proveniente de un pequeño tributario que pasa frente a las chacras, aunque en este caso normalmente sólo se siembra la chacra más productiva. Se han registrado años muy secos en los que este pequeño arroyo se secó y la siembra debió ser suspendida.

4. Cuando alguna de las chacras experimenta agotamiento del suelo entra en barbecho de dos o tres años. En este caso no se abren nuevas chacras, dados los costos de cerrar nuevos espacios. Al igual que cuando las cosechas no llegan a buen término, en estos años Reina debe comprar el maíz.

5. Se cultiva principalmente una variedad de maíz (amarillo), aunque en el pasado se sembraron otras. También se utilizan tres variedades de zapallo (angola, de tronco y criollo) y papa, un cultígeno resistente a las heladas. En la chacra más productiva se siembran todos los cultígenos juntos, mientras que la secundaria sólo se emplea para zapallo, dada la pobreza del suelo.

6. Los elementos de riesgo principales son las tormentas de granizo, las heladas y las sequías. Las primeras son muy frecuentes en Pampa de Achala y en ocasiones han dañado sus cultivos. Por su parte las heladas son altamente destructivas, en especial debido a que al encontrarse en un fondo de quebrada se manifiestan con particular intensidad. Con respecto a las sequías, como mencionamos, pueden implicar que una o ambas chacras no puedan ser trabajadas

\section{CASOS ARQUEOLÓGICOS}

\section{Norte del Valle de Punilla}

Se realizaron prospecciones intensivas en las cuencas de los arroyos Las Cuevas y Las Chacras (Figuras 3 y 4), en áreas de aprox. 18 km², dirigidas a la identificación y registro de los sitios arqueológicos presentes. Las mismas implicaron el recorrido sistemático de quebradas, afloramientos rocosos, cortes del terreno, superficies expuestas al laboreo agrícola y zanjones. Los sitios localizados fueron posicionados con GPS, fotografiados y ubicados en la cartografía regional (escala 1:50000). Asimismo, se efectuaron recolecciones sistemáticas de materiales superficiales, planos de aleros y áreas de molienda.

Los sitios identificados presentan una marcada variabilidad en cuanto a sus condiciones de emplazamiento (a cielo abierto o en abrigo rocoso), tamaño, situación topográfica, control 


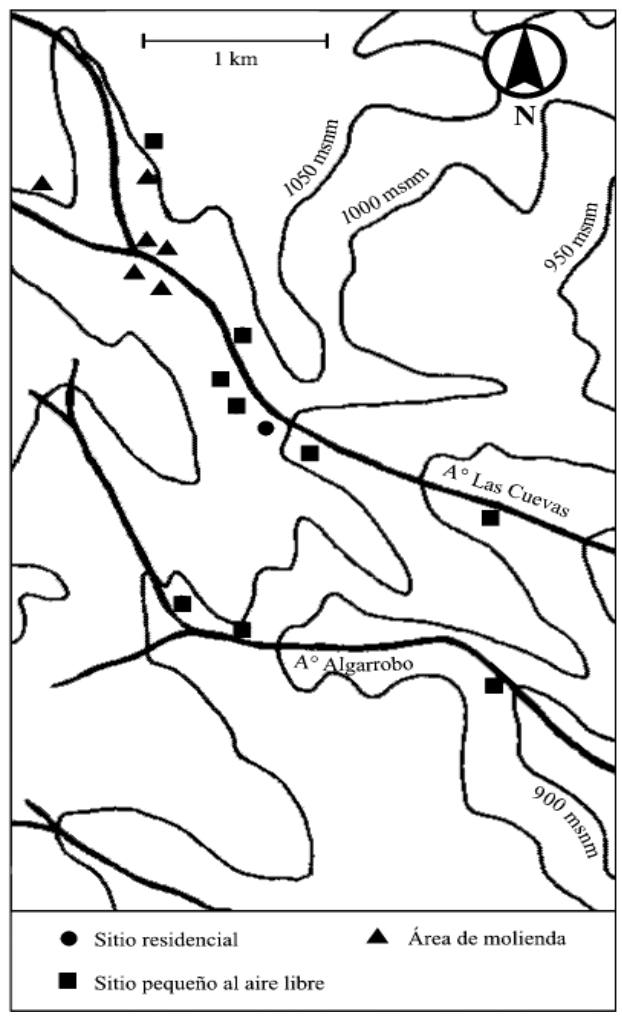

Figura 3. Sitios arqueológicos Arroyo Las Cuvas, Punilla.

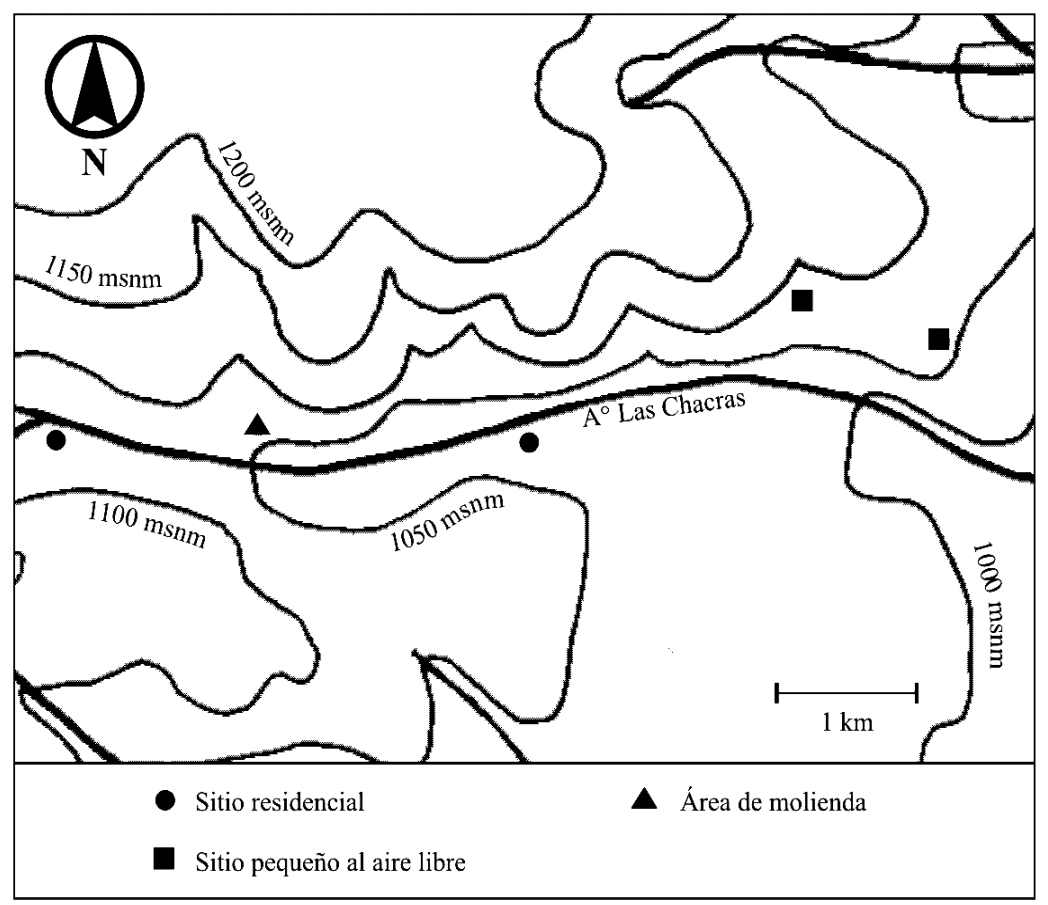

Figura 4. Sitios arqueológicos Arroyo Las Chacras, Punilla 
visual y composición artefactual. En la mayor parte de los casos, esta variabilidad no puede explicarse en base a factores cronológicos, sino que denotarían diferencias de orden funcional dentro de un mismo sistema de asentamiento. Una situación similar ha sido reseñada para el caso de la porción meridional del valle (Berberián y Roldán 2001; Roldán 1998; Roldán y Pastor 1997, 1999).

En líneas generales, los asentamientos fueron discriminados en cuatro grandes grupos:

1- Bases residenciales de actividades múltiples. Sitios a cielo abierto emplazados en terrenos potencialmente cultivables de más de 10 hectáreas, con cursos de agua de régimen anual. A nivel superficial se caracterizan por tener una alta densidad y riqueza artefactual, que incluye instrumentos de molienda fijos y móviles (conanas y morteros), manos, artefactos y desechos líticos, instrumentos agrícolas (azadas líticas), fragmentos cerámicos, artefactos óseos, torteros, entierros, etc. Dicho registro arqueológico estaría indicando mayores tiempos de permanencia y la realización de actividades múltiples propias de los espacios residenciales, como el almacenamiento, procesamiento y consumo de alimentos, manufactura lítica y textil, inhumaciones, etc. En ocasiones, se asocian con ocupaciones en abrigos rocosos, que probablemente funcionaron como viviendas o áreas de actividades específicas. Para el caso del Arroyo Las Chacras, son ejemplos de estos sitios C.Pun.39 y Las Chacras 5. El análisis preliminar de los materiales obtenidos en la excavación del primero de ellos permitió comprobar dicha asignación funcional (Argüello de Dorsch 1983; Medina 2004). La identificación de restos carbonizados de Phaseolus vulgaris y P. lunatus (n.v. poroto) junto con azadas líticas corrobora el carácter agrícola aldeano de estos sitios. Dos dataciones sobre muestras de carbón permitieron posicionar al sitio entre $854 \pm 34$ años ${ }^{14} \mathrm{C}$ AP (AA62338) y $716 \pm 34$ años ${ }^{14} \mathrm{C} \mathrm{AP} \mathrm{(AA62339).}$

2- Viviendas aisladas en abrigos rocosos. Incluye un único caso, conocido como "Cueva de los Indios", ubicada sobre el Arroyo Las Cuevas. La evidencia arqueológica obtenida durante su excavación indica la realización de una amplia gama de actividades típicas de los contextos residenciales (González 1949). Este hecho, sumado a las condiciones óptimas de su emplazamiento, lleva a pensar que el alero fue una vivienda ocupada de manera relativamente estable por un reducido número de personas. Sitios de características similares fueron descriptos para el sur del Valle de Punilla (Berberián y Roldán 2001; Nielsen y Roldán 1991; Roldán y Pastor 1999).

1- Áreas de molienda. Sitios al aire libre emplazados a orillas de los principales cursos de agua. Contienen a nivel superficial artefactos de molienda fijos, conanas y morteros, con predominio de estos últimos. Pueden citarse como ejemplos el área de molienda 3 de C.Pun.39 y Las Chacras 6, sobre el arroyo Las Chacras, y Las Cuevas 7 (LC7), LC8, LC9, LC11, LC12 y Moradillo 1, sobre el Arroyo Las Cuevas y tributarios.

2- Sitios a cielo abierto de pequeñas dimensiones. Se trata de sitios emplazados en terrenos sedimentarios aptos para la agricultura, que normalmente no superan las dos hectáreas. Exhiben diversidad topográfica, sobre todo con relación a su altitud, pendiente y orientación. En todos los casos la evidencia arqueológica a nivel superficial es escasa, limitándose a unos pocos desechos de talla, fragmentos cerámicos e instrumentos de molienda. Se considera a estos sitios como posibles campos de cultivo disociados de los espacios residenciales. Corresponden a este grupo LC1, LC2, LC4, LC6, LC10, Algarrobo 1 (A1), A2 y A3, en el sector del Arroyo Las Cuevas, y Las Chacras 3 (LCh3) y LCh4 en el Arroyo Las Chacras. Este tipo de sitios aún no ha sido trabajado sistemáticamente. Sin embargo, los materiales obtenidos durante la excavación de LC4 por Alberto Rex González -que incluyen 


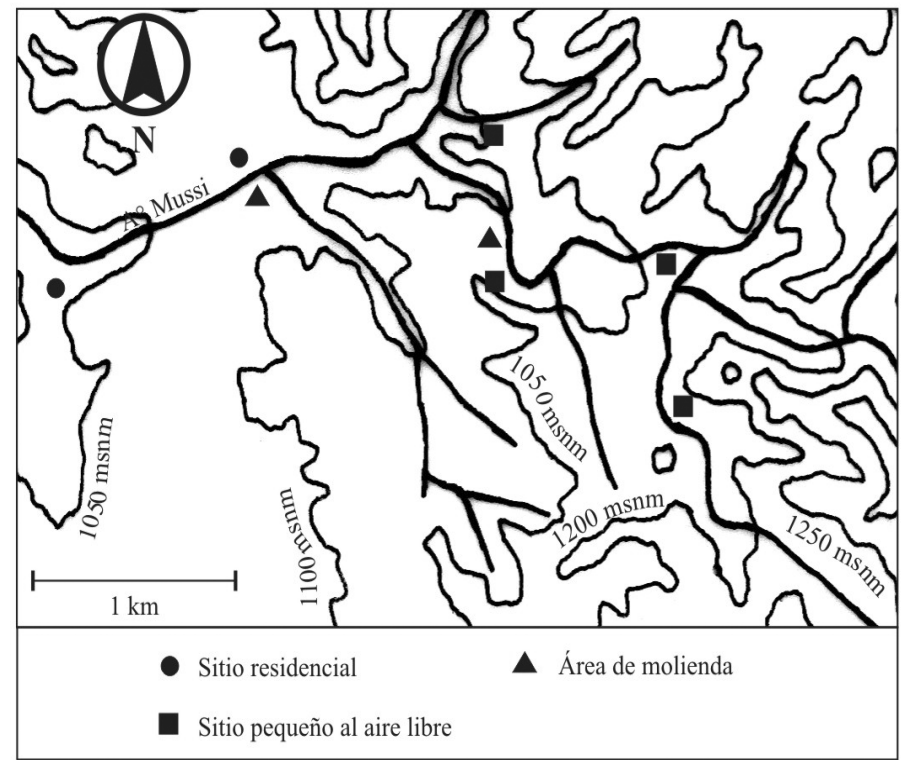

Figura 5. Sitios Arqueológicos. Cerro La Higuerita, Salsacate

un entierro- parecen sugerir la presencia de una vivienda aislada, probablemente de tipo casa-pozo (González 1949: 468) .

\section{Valle de Salsacate}

A modo de ejemplo de las situaciones arqueológicas reconocidas en el Valle de Salsacate, se presentan los resultados de las prospecciones realizadas en dos áreas de dimensiones

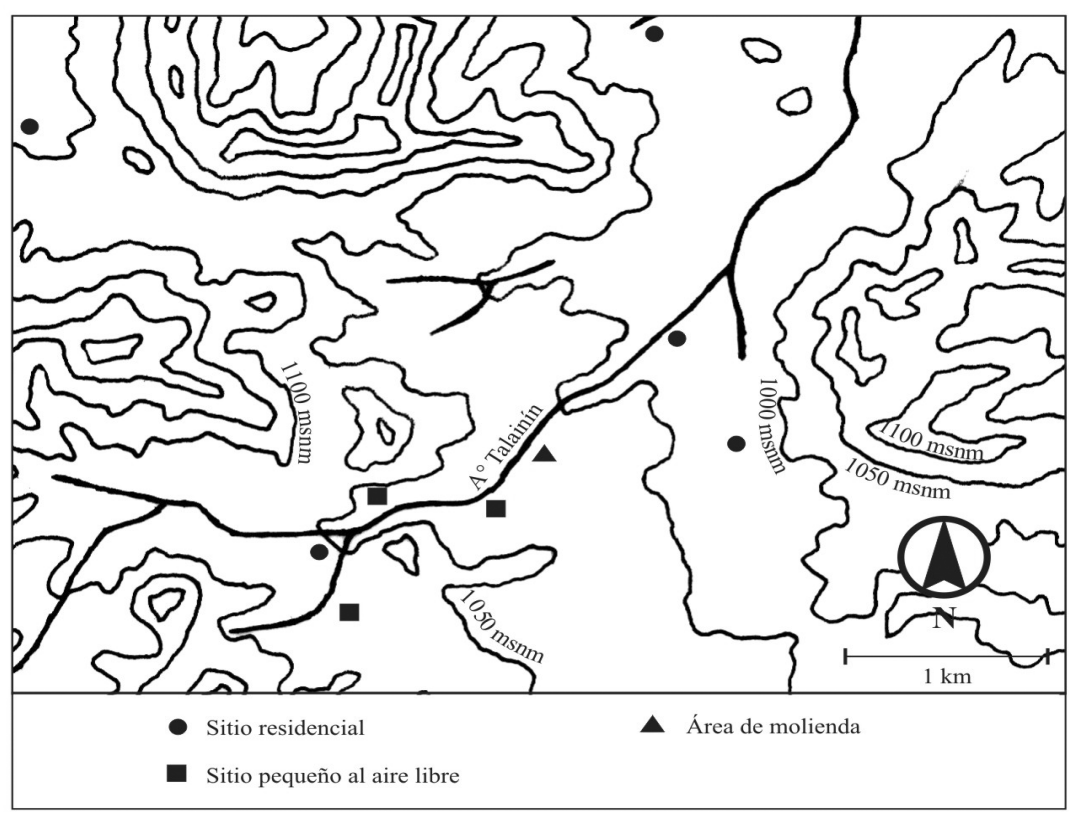

Figura 6. Sitios Arqueológicos Cañada de Salas y Alto de la Cruz, Salsacate. 
similares a las reseñadas en el caso anterior. La primera, denominada "Cerro La Higuerita" (Figura 5), se ubica en el sector sudoriental del valle, mientas que la segunda, "Cañada de Salas", se localiza en la porción sudoccidental (Figura 6).

Los sitios arqueológicos identificados exhiben patrones de estructuración y distribución similares a los de la porción septentrional del Valle de Punilla, descriptos en la sección anterior, así como a los del sector meridional (Berberián y Roldán 2001; Roldán 1998; Roldán y Pastor 1997, 1999). Estas propiedades del registro arqueológico regional parecen comunes a diferentes valles del sector central de las Sierras Centrales.

Por todo lo expuesto, para la descripción de los patrones registrados en el Valle de Salsacate se emplean las mismas categorías utilizadas en la sección anterior para el norte del Valle de Punilla.

En primer lugar, las bases residenciales se localizan en grandes terrenos potencialmente cultivables, con asociación a cursos de agua. Corresponden a este grupo los sitios Arroyo de Mussi 5 (AM5) y AM7, en Cerro La Higuerita, y Alto de la Cruz 2 (AC2), Cañada de Salas 1 (CS1), CS2, Las Totoras y La Aguadita, en Cañada de Salas. Asociados al sitio AC2 se ubican dos ocupaciones en abrigos rocosos (AC3 y AC4), que podrían constituir áreas de actividades específicas próximas a la base residencial.

En las áreas de Cañada de Salas y Cerro La Higuerita no se registraron sitios atribuibles a la segunda categoría, viviendas aisladas en abrigos rocosos, aunque éstos se encuentran presentes en otros sectores del valle (Pastor 2002a).

En cuanto a las áreas de molienda, se detectaron casos integrados en bases residenciales (AC2, CS2, Las Totoras), así como sitios separados de aquellas (AM3 y AM6). En este punto es interesante destacar la presencia del sitio Arroyo Talainín 2 (AT2), en Cañada de Salas, que comprende un pequeño abrigo rocoso asociado a una gran área de molienda integrada por 56 instrumentos fijos del tipo mortero. Las excavaciones efectuadas dentro y fuera del abrigo indican la importancia de la realización de actividades de procesamiento y consumo de alimentos a escala extradoméstica (Pastor 2004).

Por último, se reconocieron numerosos casos correspondientes a la categoría "sitios a cielo abierto de pequeñas dimensiones", que presentan características muy similares a los reconocidos en el norte del Valle de Punilla. Ellos son Arroyo Titiorco 1, AM1, AM2 y AM4, en Cerro La Higuerita, y AC1, AC5 y AT1, en Cañada de Salas.

\section{DISCUSIÓN}

En la Figura 2 se puede observar que a cada base residencial de agricultores actuales le corresponde un número variable de chacras. Las mismas se disponen a lo largo de una gradiente altitudinal, aprovechando distintos microsectores del espacio. Esta diversificación espacial no parece responder solamente a la escasez de terrenos cultivables que caracteriza a la topografía serrana, sino que representa una estrategia que permite amortiguar las variaciones en la productividad de los recursos agrícolas.

Dada la cercanía temporal inmediata al período prehistórico en estudio, se asume que los factores de riesgo agrícola que actúan en el presente son los mismos que operaron en el 
pasado. En consecuencia, se puede suponer que las comunidades productoras de alimentos debieron desarrollar estrategias dirigidas a minimizar la impredecibilidad anual de las precipitaciones, las plagas, el granizo y las heladas fuera del período normal.

En las Figuras 3, 4, 5 y 6 se puede observar que los ejemplos arqueológicos del Valle de Salsacate y porción norte del Valle de Punilla parecen repetir el mismo patrón espacial que los casos actuales. A cada sitio arqueológico interpretado como base residencial, ya sea a cielo abierto o aprovechando un abrigo rocoso, le corresponden otros asentamientos pequeños categorizados como sitios a cielo abierto de pequeñas dimensiones. Esta situación nos lleva a pensar que los grupos en estudio enfrentaron el riesgo agrícola mediante la dispersión de sus campos de cultivo, en lugar de concentrarlos en un único punto del paisaje. De acuerdo al patrón espacial de los casos señalados, las probables chacras también eran emplazadas en una amplia diversidad de microsectores del paisaje (fondos de valle, cañadas laterales, laderas de los cerros, cotas altas, cerca del río, quebradas protegidas, etc.).

La evidencia superficial registrada en estos últimos sitios (escasos desechos de talla, fragmentos cerámicos, instrumentos de molienda) sumado al hallazgo de un entierro en excavación, permiten hipotetizar la presencia de alguna vivienda aislada, ocupada por personas involucradas en el laboreo, cuidado y cosecha de los cultivos. Sin embargo, este punto debe ser apoyado con la realización de excavaciones estratigráficas que permitan contar con mayor información.

La instalación de campos de cultivo dispersos pudo formar parte de una estrategia de diversificación dentro de las prácticas agrícolas. Aún no se cuenta con datos acerca de la existencia de policultivos, tanto con relación a las diversas especies cultivadas como a sus variantes fenotípicas, aunque debe recordarse que las crónicas españolas mencionan algunas especies. Hacia este punto se orientan trabajos arqueobotánicos en curso, mediante el estudio de macro y microrestos vegetales (López, comunicación personal 2005). El análisis de los materiales recuperados en las recientes excavaciones de los sitios Río Yuspe 11, Río Yuspe 14, Arroyo Talainín 2, C.Pun.39, Los Algarrobos 1, Las Chacras 2, Puesto La Esquina 1 y Arroyo Tala Cañada 1 seguramente arrojará resultados de interés.

En síntesis, la información etnohistórica, arqueológica y etnográfica apuntan hacia la existencia de un sistema agrícola de pequeña escala, sometido a factores de riesgo ambiental, el cual habría sido enfrentado a través de la dispersión de chacras y el uso de policultivos.

Con relación a este punto debe señalarse el carácter limitado de los cálculos demográficos basados en la extensión de los terrenos potencialmente cultivables, propuestos para el sector norte de las Sierras de Córdoba (Laguens 1999). En estos trabajos se asume una explotación agrícola "máxima y óptima", que empleó la totalidad de terrenos disponibles. Como hemos visto, este supuesto resulta difícil de sostener, ya que ninguna evidencia permite vislumbrar este tipo de explotación. En consecuencia, los cálculos no reflejarían adecuadamente la realidad y el número estimado de habitantes por kilómetro cuadrado se reduciría drásticamente, así como el porcentaje de la dieta cubierto por la actividad agrícola.

Por otra parte, a partir de la información obtenida de las entrevistas con Don Jesús Reina, se puede concluir que la agricultura de pequeña escala es una actividad practicable en los ambientes serranos de altura. No obstante, se perciben diferencias con las prácticas agrícolas 
desarrolladas en los valles. Básicamente, los niveles de pérdida son mayores, circunstancia que determina que la agricultura ocupe un rol de menor importancia y que la inversión de trabajo sea muy limitada. En este sentido, no se desarrollan estrategias para enfrentar el riesgo ambiental, sino que simplemente se siembra ante la posibilidad de obtener cosechas, situación que muchas veces se ve frustrada.

A pesar de este carácter secundario, la posibilidad de practicar una agricultura de pequeña escala conduce a replantear el rol asignado a los ambientes serranos de altura en el marco de las estrategias de subsistencia-asentamiento desarrolladas por las comunidades productoras de alimentos.

La mayor parte de los sitios registrados hasta el presente en estos sectores representan ocupaciones discretas, que han sido relacionadas con la existencia de movimientos logísticos originados en las bases residenciales localizadas en los valles, los cuales tuvieron por objetivo la captura de especies faunísticas de alto rendimiento propias de este ambiente (i.e. guanacos -Lama guanicoe- y venados de las pampas -Ozotoceros bezoarticus-; Berberián y Roldán 2001; Pastor 2000). Sin embargo, existen sitios que parecen haber sido objeto de un uso que implicó la realización de un mayor número de tareas, seguramente vinculadas a un mayor tiempo de permanencia (por ejemplo, Río Yuspe 14; Pastor 2002b). La mayor permanencia en los ambientes serranos de altura cobraría coherencia en un contexto en el cual existió algún manejo de camélidos domésticos, cuya presencia ha sido recientemente sugerida por estudios osteométricos y otras líneas de evidencias (Pastor y Medina 2003a).

En estos casos, no puede descartarse la realización de cultivos de pequeña escala, tal como ocurre en la actualidad. En este sentido, además de Río Yuspe 14, es sugestiva la presencia de sitios pequeños al aire libre, similares a los "sitios a cielo abierto de pequeñas dimensiones", comunes en los valles serranos. Estos sitios (Casa de Reina 3, Río Yuspe 3, Hueco de Anselmo 1, El Plumerillo 2) se ubican en quebradas protegidas en el sector norte de la Pampa de Achala y sur de la Pampa de San Luis (Pastor 2002a). De acuerdo a las características de su contenido arqueológico (desechos e instrumentos líticos, fragmentos cerámicos, instrumentos de molienda), parece probable que hayan albergado algunas viviendas, posiblemente ocupadas por personas dedicadas al cuidado de animales domésticos quienes, habitualmente, pudieron instalar pequeños campos de cultivo en las proximidades. Sin dudas, el estudio sistemático de estos sitios aportará información de utilidad para comprender la diversidad de usos de los que fueron objeto los ambientes de altura durante el período prehispánico tardío.

\section{CONSIDERACIONES FINALES}

En este trabajo hemos intentado comenzar a discutir algunos aspectos de la producción agrícola durante el período prehispánico tardío en las Sierras de Córdoba, a partir de información etnográfica actual, de documentación etnohistórica y de la distribución espacial de sitios arqueológicos.

Se propone la existencia de un sistema agrícola de pequeña escala, caracterizado por la instalación de campos de cultivo dispersos, que representaría una estrategia destinada a enfrentar numerosos factores de riesgo, como las tormentas de granizo, las heladas tempranas o tardías y las plagas de langostas. Este tipo de estrategia ha sido ampliamente documentado 
en casos etnográficos y arqueológicos de la zona andina (Browman 1987; Goland 1993; Muscio 2004; Winterhalder et al. 1999), así como en distintos puntos del mundo (Halstead y O’Shea 1989; Winterhalder y Goland 1997; Winterhalder et al. 1999).

En la sierras de Córdoba la agricultura de pequeña escala se habría practicado en los diferentes valles serranos, donde constituiría la actividad económica principal, aunque no se descarta su presencia en los ambientes de altura, donde su carácter sería secundario y, posiblemente, estaría llevada a cabo por personas instaladas en forma estacional.

Indudablemente, deben sumarse a la discusión nuevas líneas de evidencia, fundamentalmente derivadas de estudios arqueobotánicos y de la excavación de los denominados "sitios a cielo abierto de pequeñas dimensiones".

Hasta hace pocos años la mayor parte de los aspectos relacionados con las estrategias de subsistencia de la población aborigen se trataban a partir de líneas de evidencia indirectas. El inicio de estudios arqueofaunísticos ha permitido desarrollar una visión acerca de las actividades cinegéticas y pastoriles implementadas por estos grupos (Medina 2001, 2002; Pastor y Medina 2003b), aunque el conocimiento arqueológico de actividades como la agricultura y la recolección es prácticamente inexistente.

Por estemotivo, este trabajo sólo pretendeiniciar la discusión, cuestión queconsideramosnecesaria, aún cuando esperamos contar, en el mediano plazo, con mayor información arqueológica.

\section{AGRADECIMIENTOS}

Deseamos expresar nuestro reconocimiento al Dr. Eduardo Berberián, quien se encargó de la supervisión general de las tareas y de efectuar valiosas sugerencias durante la elaboración del trabajo. Timothy Jull (NSF Arizona AMS Facility) y la National Science Foundation (Grant EAR0115488) colaboraron en la realización de los fechados radiocarbónicos de C.Pun.39. Germán Figueroa, Bruno Catania, Catriel Greco, Ana Liboreiro, Natalia Bidart, Ezequiel Gilardengui, Pablo Giorno y Laura López participaron activamente en los trabajos de campo, mientras que las familias de Benito Arias, Julián Cataldo, Marcos Oliva, Jesús Reina y Rosa de López aportaron importante información acerca de sus prácticas agrícolas. Los autores somos los únicos responsables de los conceptos vertidos en el trabajo.

\section{BIBLIOGRAFÍA}

Argüello de Dorsch, E.

1983. Investigaciones arqueológicas en el Departamento Punilla (Provincia de Córdoba República Argentina). Sitio: C. Pun. 39. Comechingonia 1: 41-60.

Berberián, E.

1999. Sierras Centrales. Nueva Historia de la Nación Argentina (ed. por la Academia Nacional de la Historia), pp. 135-158. Editorial Planeta, Buenos Aires.

Berberián, E. y F. Roldán

2001. Arqueología de las Sierras Centrales. Historia Argentina Prehispánica (ed. por E. Berberián y A. Nielsen), pp. 635-691. Editorial Brujas, Córdoba. 
Bixio, B. y E. Berberián

1984. Etnohistoria de la región de Potrero Garay (Pcia. de Córdoba - Rep. Argentina). Comechingonia 3: 11-46.

Bousman, C.

1993. Hunter-gatherer adaptation, economic risk and tool design. Lithic Technology 18, No 1 y 2.

Browman, D.L.

1987. Agro-Pastoral Risk management in the Central Andes. Research in Economic Anthropology 8: $171-200$

Bucher, E. y J. Abalos

1979. Fauna. Geografia Física de la Provincia de Córdoba (ed. por J. Vázquez, R. Miatello y M. Roqué), pp. 369-434. Editorial Boldt, Buenos Aires.

Capitanelli, R.

1979. Clima. Geografía Física de la Provincia de Córdoba (ed. por J. Vázquez, R. Miatello y M. Roque), pp. 45-138. Editorial Boldt, Buenos Aires.

Cashdan, E. (ed.)

1990. Risk and uncertainly in tribal and peasant economies. Westwiew Press, San Francisco.

Goland, C.

1993. Cultivating Diversity: Field Scattering as Agricultural Risk Management in CuyoCuyo, Department of Puno, Perú. Culture and Agriculture 45-46: 8-13.

González, A.

1949. Nota sobre la arqueología de Pampa de Olaen (Córdoba). Notas del Museo de La Plata, Tomo XIV, Antropología No 56: 463-503.

Halstead, P. y J. O’Shea (eds.) 1989. Bad Year Economic: Cultural Responses to Risk and Uncertainty. Cambridge University Press, Cambridge.

Servicio Meteorológico Nacional 1985. Heladas. Boletín 17. Fuerza Aérea Argentina, Buenos Aires.

Hordij, H., S. Bordón y R. Sassone

1996. Análisis del a marcha anual de las cartas isoceráunicas mensuales en la República Argentina. Trabajo presentado en el VII Congreso Argentino de Meteorología y VII Congreso Latinoamericano e Ibérico de Meteorología. Buenos Aires.

Laguens, A.

1999. Arqueología del contacto hispano-indígena. Un estudio de cambios y continuidades en las Sierras Centrales de Argentina. BAR, International Series 801, Oxford.

López Bellido, L.

1991. Cultivos Herbáceos. Ediciones Mundi-Prensa, Madrid 
Medina, M.

2001. La amplitud máxima de la dieta en contextos formativos tardíos del sector central de Sierras Centrales. Milenio (ed. por M. Caggiano), pp. 97-100. Chivilcoy.

2002. Arqueofaunas y tafonomía: la importancia de la caza en contextos formativos tardios del sector central de Sierras Centrales. Tesis de Licenciatura en Ciencias. Antropológicas. Facultad de Filosofía y Letras. Universidad de Buenos Aires, Buenos Aires.

2004. Diversificación económica y uso del espacio entre las poblaciones productoras de alimentos del norte de Punilla y Llanura Noroccidental (Córdoba, Argentina). Informe presentado al CONICET. Córdoba, Ms.

Muscio, $\mathrm{H}$.

2004. Dinámica Poblacional y Evolución durante el Período Agroalfarero Temprano en el Valle de San Antonio de los Cobres, Puna de Salta, Argentina. Tesis Doc.. F.FyL. UBA, Buenos Aires.

Nielsen, A. y F. Roldán

1991. Asentamientos satélites y asentamientos agrícolas permanentes: el caso "El Fantasio" (Depto. Punilla - Córdoba). Comechingonia 7: 65-75.

Papadakis, J.

1956. Informe ecológico sobre la Provincia de Córdoba. G.A.E.A. 10: 103-156.

Pastor, S.

2000. Producción lítica en Puesto Maldonado 3 y La Hoyada 6 (Pampa de Achala). Una aproximación a las estrategias tecnológicas de las comunidades productoras de alimentos de la región serrana de Córdoba. Tesis de Lic en Historia. F.F.y H. Universidad Nacional de Córdoba, Córdoba.

2002a. Subsistencia, movilidad y tecnología de las comunidades productoras de alimentos del Valle de Salsacate y pampas de altura adyacentes (Pcia. de Córdoba). Inf. presentado al CONICET, Cba. Ms

2002b. El sitio Río Yuspe 14 (Pampa de Achala, Córdoba). Perspectivas sobre el uso prehispánico tardío de los ambientes serranos de altura. Córdoba. Ms

2004. Las actividades de procesamiento-consumo y las relaciones intergrupales en el período prehispánico tardío de las Sierras de Córdoba. Arqueología de Arroyo Talainín 2 y Río Yuspe 11. Anuario de la Escuela de Historia 3: 99-118. Córdoba.

Pastor, S. y M. Medina

2003a. Osteometría de camélidos en sitios tardíos de las Sierras de Córdoba (Argentina). Primeras evidencias sobre la presencia de Lama glama. El manejo de los camélidos sudamericanos. (ed. por G. Mengoni Goñalons, D.E. Olivera y H.D. Yacobaccio). Grupo de Zooarqueología de Camélidos - Internacional Council of Archaeozoology, Buenos Aires. (en prensa).

2003b. Los pequeños vertebrados y la hipótesis del "Garden Hunting". Un caso prehistórico de las Sierras de Córdoba (Argentina). Córdoba. Ms.

Piana de Cuestas, J.

1992. Los indígenas de Córdoba bajo el régimen colonial (1570-1620). Dirección General de Publicaciones de la Universidad Nacional de Córdoba, Córdoba.

Roldán, F.

1998. Estructura de recursos, sistema de asentamiento y movilidad en momentos previos a la conquista hispánica en la cuenca del Río San Antonio (Pcia. de Córdoba). Trabajo presentado en las III Jornadas de Invest. en Arqueología y Etnohistoria del Centro Oeste del País. Río Cuarto. 
Roldán, F. y S. Pastor

1997. Tipos de asentamientos prehispánicos en la porción meridional del Valle de Punilla (Pcia. de Córdoba). Actas de las Jornadas de Antropología de la Cuenca del Plata, tomo III, pp. 4854. Rosario.

1999. Variabilidad ocupacional en los abrigos rocosos del sur del Valle de Punilla (Provincia de Córdoba). Actas de las Segundas Jornadas de Investigadores en Arqueología y Etnohistoria del Centro-Oeste del País, pp. 59-69. Río Cuarto.

Terzaga, A.

1963. Geografía de Córdoba. Editorial Assandri, Córdoba.

Winterhalder, B. y C. Goland

1997. An Evolutionary Ecology Perspective on Diet Choice, Risk, and Plant domestication. Peoples, plants and landscapes, Studies in paleoetnobotany (ed. por K.J. Gremilion), pp. 123-160. The University of Alabana Press, Tuscaloosa and London.

Winterhalder, B., F. Lu y B. Tucker 1999. Risk Sensitive Adaptative Tactics: Models and Evidence from Subsistence Studies in Biology and Anthpology. Journal of Archaeological Research 7 (4): 301-343. 
